\title{
¿INFLUYEN LAS EXPERIENCIAS CLIMÁTICAS ADVERSAS Y SUS CONSECUENCIAS EN LA PERCEPCIÓN QUE TIENE EL AGRICULTOR SOBRE EL CAMBIO CLIMÁTICO?
}

\author{
$\underline{\text { Julia M. Núñez-Tabales }}{ }^{a *}$ y Francisco J. Rey-Carmona ${ }^{a}$ \\ ${ }^{a}$ Departamento de Estadística, Econometría, I.O. y Organización de Empresas. Universidad de Córdoba \\ (Córdoba, España, es2nutaj@uco.es, td1recaf@uco.es)
}

\begin{abstract}
Resumen
El cambio climático conlleva un aumento de riesgos de producción en la agricultura de regadío de las zonas mediterráneas derivados, entre otros motivos, de la disminución de las lluvias, de la mayor incidencia de episodios de golpes de calor, de sequías más prolongadas y del incremento de lluvias torrenciales e inundaciones. El presente trabajo persigue como objetivo analizar las percepciones acerca del cambio climático de los agricultores de regadío de la Cuenca Hidrográfica del Guadalquivir. Para ello se entrevistó personalmente a una muestra de agricultores mediante cuestionario estructurado. En función de dicha percepción se obtienen varios segmentos de agricultores entre los que se analizan si existen diferencias en su experiencia acerca de diversas manifestaciones climáticas adversas para sus cultivos acontecidas durante los últimos años. Los resultados evidencian diferencias entre los segmentos en seis de los escenarios presentados. La determinación de las percepciones de este colectivo y de los elementos que afectan a dicha percepción será un importante aspecto a considerar en el examen y evaluación de medidas o instrumentos que permitan la adaptación o mitigación del impacto del cambio climático.
\end{abstract}

Palabras clave: Cambio climático; percepciones; experiencias previas; agricultura de regadío.

\section{Introducción}

Las proyecciones de la comunidad científica señalan que en la región mediterránea el cambio climático dará lugar a un descenso de las precipitaciones, una intensificación de los períodos de sequía o un incremento de la temperatura superior al promedio mundial [Giorgi y Lionello (2008); Tuel y Eltahir (2020)].

En este escenario, el sector agrario es uno de los más vulnerables a dichos cambios, ya que pondrán en riesgo la productividad de las cosechas. Por este motivo, diversos autores han subrayado la importancia de adaptar la agricultura a las alteraciones en el clima [Iglesias et al. (2011)]. Sin embargo, el análisis de la percepción del cambio climático es un requisito imprescindible y previo a la adaptación, ya que es coherente esperar que aquel agricultor que percibe el cambio esté más predispuesto a adoptar determinadas prácticas para mitigar posibles efectos adversos asociados [Rejesus et al.(2013)].

Por consiguiente, esta investigación persigue un doble objetivo. Por un lado, analizar las percepciones sobre el cambio climático que tiene el agricultor ubicado en la Cuenca Hidrográfica del Guadalquivir (CHG) y, por otro lado, evaluar la existencia de una vinculación entre el nivel de conciencia que el agricultor tiene del cambio climático y su propia experiencia sobre manifestaciones climáticas adversas.

Existen en la literatura escasos estudios que analizan la percepción del cambio climático y su vinculación con experiencias climáticas pasadas [Myers et al. (2013); Haden et al. (2012); Niles et al. (2013)]. Nuestro estudio está diseñado para rellenar esta brecha de conocimiento existente a través de un enfoque cuantitativo.

\section{Metodología}

El estudio se llevó a cabo en explotaciones de regadío ubicadas en el marco geográfico de la CHG, escenario caracterizado por un clima típico mediterráneo. Los datos se recopilaron mediante entrevistas personales llevadas a cabo en los meses de otoño de 2018. En total se obtuvieron 204 cuestionarios completos.

Aunque la entrevista constaba de varias partes, este trabajo se centró en analizar solo determinadas cuestiones. Concretamente se analizó el grado de acuerdo del encuestado en una escala Likert - graduada del 1 (muy en desacuerdo) al 5 (muy de acuerdo) - con respecto a si el cambio climático está teniendo lugar a nivel global, por un lado, y a nivel local, por otro lado. A continuación, se le interrogó (respuesta dicotómica afirmativa/negativa) sobre su conciencia acerca de ocho posibles escenarios adversos ocurridos en su explotación durante los últimos años, produciendo daños en los cultivos: incremento de la escasez de agua para riego (Esc_1), incremento de las necesidades hídricas de los cultivos (Esc_2), deterioro de la calidad del agua (Esc_3), más lluvias torrenciales e inundaciones (Esc_4), más episodios de golpes de calor (Esc_5), disminución de horas de frío en otoño/invierno (Esc_6), mayores heladas y pedrisco (Esc_7) e incremento de plagas y enfermedades (Esc_8). 
La tabulación y el análisis de la información obtenida se ha realizado a través del diseño de una base de datos utilizando el programa SPSS 25.0. Se aplicaron diversas técnicas de análisis estadístico multivariante para obtención de los objetivos establecidos.

\section{Resultados}

En cuanto a la percepción manifestada por los agricultores acerca del cambio climático a nivel global, los resultados evidencian que más de las tres cuartas partes de los encuestados mostraron estar "de acuerdo" o "muy de acuerdo" con la afirmación relativa a que el cambio climático a nivel global está ocurriendo. Sin embargo, respecto a si el cambio climático está teniendo lugar a nivel local, los encuestados ofrecen respuestas mucho más dispersas que en el caso anterior, dado que solo algo más de la mitad reconoce estar "de acuerdo" o "muy de acuerdo" con dicha afirmación.

A continuación, se realizó un análisis preliminar para conocer si existen diferencias significativas en las puntuaciones medias otorgadas a la percepción del cambio climático (tanto a nivel global como a nivel local) entre los que sí detectaron situaciones dañinas para la explotación en los últimos años y los que no. Para ello se calculó la prueba t para muestras independientes. Los resultados obtenidos se recogen en el Cuadro 1 y ponen de manifiesto que existen diferencias significativas en las puntuaciones medias otorgadas a la percepción del cambio climático en los ocho escenarios manejados, a excepción del escenario 8 (incremento del riesgo de plagas y enfermedades) para el que no hay diferencia significativa en las puntuaciones otorgadas al cambio climático local Puede apreciarse que los que responden afirmativamente a la experiencia de un determinado escenario otorgan siempre una puntuación mayor a la percepción del cambio climático que los que responden negativamente.

Cuadro 1. Puntuaciones medias en la percepción del cambio climático a nivel global y local en función de si se han experimentado o no escenarios dañinos para la explotación en el pasado (prueba t)

\begin{tabular}{|c|c|c|c|}
\hline & & CC Global & CC Local \\
\hline \multirow{3}{*}{ Esc_1 } & Sí & 4,27 & 3,7 \\
\hline & No & 3,67 & 2,86 \\
\hline & $\mathrm{t}$ (p-value) & $3,591(0,000)^{* * *}$ & $4,374(0,000)^{* * *}$ \\
\hline \multirow{3}{*}{ Esc_2 } & Sí & 4,4 & 3,82 \\
\hline & No & 3,58 & 2,8 \\
\hline & $\mathrm{t}$ (p-value) & $5,366(0,000)^{* * *}$ & $5,599(0,000)^{* * *}$ \\
\hline \multirow{3}{*}{ Esc_3 } & Sí & 4,33 & 3,67 \\
\hline & No & 3,81 & 3,13 \\
\hline & $\mathrm{t}$ (p-value) & $3,237(0,001)^{* * *}$ & $2,727(0,007)^{* * *}$ \\
\hline \multirow{3}{*}{ Esc_4 } & Sí & 4,2 & 3,58 \\
\hline & No & 3,6 & 2,83 \\
\hline & $\mathrm{t}$ (p-value) & $3,368(0,001)^{* * *}$ & $3,717(0,000)^{* * *}$ \\
\hline \multirow{3}{*}{ Esc_5 } & Sí & 4,22 & 3,65 \\
\hline & No & 3,19 & 2,12 \\
\hline & $\mathrm{t}$ (p-value) & $4,500(0,000)^{* * *}$ & $7,215(0,000)^{* * *}$ \\
\hline \multirow{3}{*}{ Esc_6 } & Sí & 4,13 & 3,48 \\
\hline & No & 3,55 & 2,81 \\
\hline & $\mathrm{t}(\mathrm{p}$-value $)$ & $2,421(0,019)^{* *}$ & $2,502(0,015)^{* *}$ \\
\hline \multirow{3}{*}{ Esc_7 } & Sí & 4,42 & 3,69 \\
\hline & No & 3,88 & 3,23 \\
\hline & $\mathrm{t}$ (p-value) & $2,855(0,005)^{* * *}$ & $2,028(0,044)^{* *}$ \\
\hline \multirow{3}{*}{ Esc_8 } & Sí & 4,2 & 3,41 \\
\hline & No & 3,89 & 3,29 \\
\hline & $\mathrm{t}$ (p-value) & $1,906(0,058)^{*}$ & $0,582(0,561)^{\mathrm{Ns}}$ \\
\hline
\end{tabular}

Fuente: Elaboración propia.

Atendiendo a las percepciones del agricultor acerca de si el cambio climático está teniendo lugar a nivel global y a nivel local se efectuó un análisis de conglomerados no jerárquico, obteniéndose tres segmentos 
diferenciados. El primero de ellos muestra una percepción elevada del cambio climático, el tercero denota una percepción reducida y el segundo una percepción intermedia.

Para buscar diferencias en cuanto a la conciencia de los ocho escenarios adversos entre las tres categorías se realizó la prueba Chi-cuadrado. Se trabajó con los porcentajes de respuestas afirmativas para detectar las posibles diferencias.

Gráfico 1. Comparativa entre los tres segmentos de respuestas afirmativas (\%) para cada uno de los escenarios

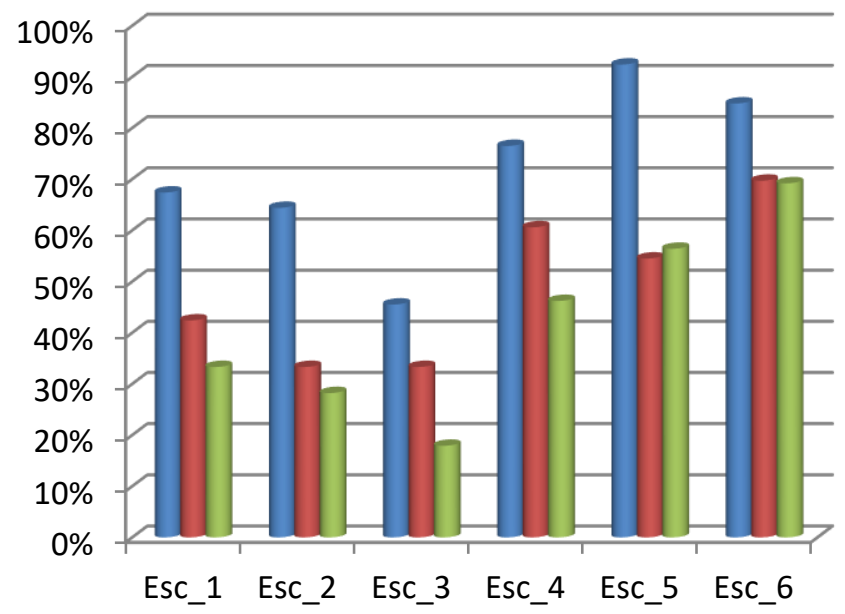

Segmento 1

Segmento 2

- Segmento 3

Esc_1 (p-valor=0,000***); Esc_2 (p-valor=0,000***); Esc_3 (p-valor=0,007 $\left.{ }^{* * *}\right) ;$ Esc_4 (p-valor=0,001 $\left.{ }^{* * * *}\right)$; Esc_5 (p-valor $\left.=0,000^{* * *}\right) ;$ Esc_6 $\left(\mathrm{p}\right.$-valor $\left.=0,034^{* *}\right)$

**** $p<0,01 ;{ }^{* *} p<0,05 ;{ }^{*} p<0,1$.

Nota: Como las variables son dicotómicas la tabla recoge el porcentaje de respuestas afirmativas.

Fuente: Elaboración propia.

Dicha prueba ofreció como resultado que existen diferencias entre las proporciones en todos los escenarios excepto en dos: el escenario 7 (mayores heladas/ pedrisco) $-\mathrm{p}$-valor $=0,334-\mathrm{y}$ el escenario 8 (incremento de plagas/ enfermedades) — p-valor=0,646- En el Gráfico 1 puede observarse la representación de los seis escenarios en los que se obtuvieron diferencias significativas entre las proporciones de los tres segmentos, siendo el primer segmento el que presenta más conciencia de los escenarios dañinos para sus cultivos (experiencias previas). Los porcentajes afirmativos de los segmentos segundo y tercero se encuentran muy por debajo del primer segmento y mucho más próximos entre sí.

\section{Conclusiones}

El cambio climático está afectando negativamente al sector agrícola en regiones mediterráneas, especialmente a la agricultura de regadío. No obstante, antes de examinar si los agricultores tomarán medidas para la adaptación y mitigación de dichos cambios, es preciso comprender cómo este colectivo percibe los cambios en el clima y qué elementos lo determinan.

La obtención en este estudio de percepciones del cambio climático a un nivel global diferentes a las percibidas a un nivel local, ha permitido agrupar a los agricultores analizados en tres categorías o niveles de percepción (máximo, intermedio y mínimo). Asimismo, se ha detectado una vinculación entre los niveles mencionados y las experiencias pasadas con escenarios climáticos adversos (excepto en dos de los escenarios manejados). Por consiguiente, experiencias climáticas adversas pasadas pueden utilizarse como variables proxy a la hora de investigar la percepción que tiene el agricultor sobre el cambio climático, es decir, no resulta imprescindible preguntarle directamente por el cambio climático en sí, dado que se ha observado una vinculación entre determinadas experiencias y el nivel de percepción del cambio climático.

\section{Agradecimientos}

Los autores agradecen la financiación parcial de esta investigación a la Consejería de Economía, Conocimiento, Empresas y Universidad de la Junta de Andalucía y al Fondo Europeo de Desarrollo Regional (FEDER) a través del proyecto de investigación FINAGUA (UCO1264548). Estas instituciones de financiación no participaron en la realización de la investigación ni en la preparación del documento. 


\section{Bibliografía}

Giorgi, F., y Lionello, P. (2008). "Climate change projections for the Mediterranean region". Global and Planetary Change, 63(2-3): 90-104.

Haden, V.R., Niles, M.T., Lubell, M., Perlman, J. y Jackson, L.E. (2012). "Global and local concerns: what attitudes and beliefs motivate farmers to mitigate and adapt to climate change?". PLOS ONE, 7: e52882.

Iglesias A., Mougou R., Moneo M. y Quiroga S. (2011). "Towards adaptation of agriculture to climate change in the Mediterranean". Regional Environmental Change, 11(1): 159-166.

Myers, T. A., Maibach, E. W., Roser-Renouf, C., Akerlof, K., y Leiserowitz, A.A. (2013). "The relationship between personal experience and belief in the reality of global warming". Nature Climate Change, 3(4): 343-347.

Niles, M.T., Lubell, M. y Haden, V. R. (2013). "Perceptions and responses to climate policy risks among California farmers". Global Environmental Change, 23(6): 1752-1760.

Rejesus, R.M., Mutuc-Hensley, M., Mitchell, P.D., Coble, K.H. y Knight, T. O. (2013). "US agricultural producer perceptions of climate change". Journal of Agricultural and Applied Economics, 45: 701-718.

Tuel, A., y Eltahir, E. A. B. (2020). "Why is the Mediterranean a climate change hot spot?". Journal of Climate, 33(14): 5829-5843. 